\title{
Bovine Tuberculosis in a Nebraska Herd of Farmed Elk and Fallow Deer: A Failure of the Tuberculin Skin Test and Opportunities for Serodiagnosis
}

\author{
W. Ray Waters, ${ }^{1}$ Gary E. Stevens, ${ }^{2}$ Mark A. Schoenbaum, ${ }^{3}$ Kathy A. Orloski, ${ }^{3}$ \\ Suelee Robbe-Austerman, ${ }^{4}$ N. Beth Harris, ${ }^{4}$ S. Mark Hall, ${ }^{4}$ Bruce V. Thomsen, ${ }^{4}$ \\ Arach J. Wilson, ${ }^{4}$ Roger E. Brannian, ${ }^{4}$ Jeffrey T. Nelson, ${ }^{4}$ Shawn Schafer, ${ }^{5}$ Javan Esfandiari, ${ }^{6}$ \\ Meghan Dutton, ${ }^{6}$ Rena Greenwald, ${ }^{6}$ and Konstantin P. Lyashchenko ${ }^{6}$ \\ ${ }^{1}$ National Animal Disease Center, Agricultural Research Service, United States Department of Agriculture (USDA), \\ Ames, IA 50010, USA \\ ${ }^{2}$ National Veterinary Services Laboratories (NVSL), Animal and Plant Health Inspection Service (APHIS), USDA, \\ Lincoln, NE 68516, USA \\ ${ }^{3}$ NVSL, APHIS, USDA, Fort Collins, CO 80526-8117, USA \\ ${ }^{4}$ NVSL, APHIS, USDA, Ames, IA 50010, USA \\ ${ }^{5}$ North American Deer Farmers Association, Lake City, MN 55041, USA \\ ${ }^{6}$ Chembio Diagnostic Systems, Inc., Medford, NY 11763, USA
}

Correspondence should be addressed to W. Ray Waters, ray.waters@ars.usda.gov

Received 10 January 2011; Accepted 20 February 2011

Academic Editor: Michael D. Welsh

Copyright ( $) 2011$ W. Ray Waters et al. This is an open access article distributed under the Creative Commons Attribution License, which permits unrestricted use, distribution, and reproduction in any medium, provided the original work is properly cited.

\begin{abstract}
In 2009, Mycobacterium bovis infection was detected in a herd of 60 elk (Cervus elaphus) and 50 fallow deer (Dama dama) in Nebraska, USA. Upon depopulation of the herd, the prevalence of bovine tuberculosis (TB) was estimated at $\sim 71-75 \%$, based upon histopathology and culture results. Particularly with elk, gross lesions were often severe and extensive. One year ago, the majority of the elk had been tested for TB by single cervical test (SCT), and all were negative. After initial detection of a tuberculous elk in this herd, 42 of the 59 elk were tested by SCT. Of the 42 SCT-tested elk, 28 were TB-infected with only $3 / 28$ reacting upon SCT. After SCT, serum samples were collected from the infected elk and fallow deer from this herd at necropsy and tested by three antibody detection methods including multiantigen print immunoassay, cervidTB STAT-PAK, and dual path platform VetTB (DPP). Serologic test sensitivity ranged from 79 to $97 \%$ depending on the test format and host species. Together, these findings demonstrate the opportunities for use of serodiagnosis in the rapid detection of TB in elk and fallow deer.
\end{abstract}

\section{Introduction}

Farmed deer represent a significant alternative livestock industry with numbers exceeding 2 million in New Zealand, 1 million in China, 500,000 in the US, 400,000 in Russia, and 100,000 in Canada [1]. Farmed deer are exposed to various other livestock and to free-ranging wildlife and are moved between herds and across borders. Thus, there is an increased risk of the spread of infectious disease among and between farmed deer, traditional livestock, and free-ranging wildlife. In addition, many deer are kept in parks, preserves, and private estates for hunting, zoological, and aesthetic purposes. Intensive management promotes the spread of infectious diseases in these populations.

Mycobacterium bovis, a member of the M. tuberculosis complex, has a wide host range as compared to other species in this disease complex, is infectious to humans, and is the species most often isolated from tuberculous cattle. Free-ranging and captive deer are implicated in the spread of $M$. bovis to cattle [2-4] and to humans [5-8]. Spread of bovine TB in captive herds within the US is strongly linked to interherd and cross-country movement 
of infected deer [3]. From 1991 to 2003, M. bovis was detected in 43 captive cervid herds [9]. The 1991 outbreak of bovine TB in captive elk (Cervus elaphus) encouraged the US government and cervid industry leaders to draft uniform methods and rules (UMR) for eradication of bovine TB in captive cervids, initially published in 1994 [3] and subsequently revised in 1999. More recently (2004-2010), $M$. bovis has been detected in 3 captive cervid farms in Indiana (elk and various other deer species), 4 captive white-tailed deer herds in Michigan, 1 captive herd in New York (red deer Cervus elaphus) and fallow deer (Dama dama), and 1 captive herd in Nebraska (present study) [10-12]. Additionally, molecular strain typing of $M$. bovis isolated from cattle in South Dakota, Nebraska, Indiana, and Kentucky matched the predominant strain of $M$. bovis in US captive cervids, suggesting transmission of $M$. bovis from captive cervids to cattle in these cases. Thus, TB in captive cervids continues to pose a significant health and regulatory concern, both for the captive cervid industry as well as for cattle producers.

In the US, farmed deer are primarily monitored by skin test and rarely by slaughter surveillance. The US program requires a negative skin test for interstate transport and includes a voluntary herd accreditation program; however, few farmed deer owners have participated in the latter. Potential reasons for low participation include inadequate handling facilities, injury and mortality resulting from handling events, poor perception by owners of the specificity of skin testing in cervids, and decreased interstate movement due to chronic wasting disease-associated restrictions in the US. For cervid producers, a blood-based TB test for initial surveillance may increase participation. Recent studies have demonstrated the potential of emerging antibody-based detection assays for use in cervids [13-18].

The present report describes the diagnosis of M. bovis infection in a captive elk and fallow deer farm with a high prevalence of disease. In particular, details on the case history, standard diagnostic techniques (i.e., slaughter surveillance, skin test, necropsy, histology, and mycobacterial culture), and emerging serologic methods are provided.

\section{Materials and Methods}

2.1. Herd History. The herd consisted of 50 fallow deer and 60 elk (includes 59 elk involved in the investigation and the TB index case detected in January, 2009). The elk herd was regularly tested by SCT as required by the TB herd accreditation program [19]. Fallow deer were not tested by SCT primarily due to the owner's concern of handlingassociated injuries. Once the index case had been indentified, as many animals as possible ( 48 fallow deer and 52 elk) were examined postmortem for TB. Samples were collected for histopathology and culture only from those animals that had gross lesions suggestive of TB.

2.2. Single Cervical Skin Test (SCT). The SCT was applied according to the USDA bovine TB eradication, $\operatorname{UMR}[3,19]$.

2.3. Isolation and Identification of Mycobacteria Spp. Tissues were processed for the isolation of $M$. bovis as described previously [20] using a combination of the BACTEC 460 radiometric system, BACTEC Mycobacteria Growth Indicator Tube (MGIT) 960 system (Becton Dickinson and Company, Sparks, MD, USA), and 4 tubes of solid media. Solid media included 2 tubes of $7 \mathrm{H} 11$ supplemented with OADC, pyruvate, calf serum, and lysed sheep blood (National Veterinary Services Laboratories, Ames, IA, USA) and 1 tube each of 7H10 supplemented with OADC, pyruvate (National Veterinary Services Laboratories, Ames, IA, USA), and Mycobactesel LJ (Becton Dickinson and Company, Sparks, MD, USA). Isolates of $M$. bovis were identified by a combination of Ziehl-Neelsen acid-fast staining, nucleic acid probes (AccuProbe, Gen-Probe, San Diego, CA, USA), and spoligotyping (Ocimum Biosolutions Ltd., Hyderabad, India). Identification of atypical Mycobacteria spp. was by $16 \mathrm{~S}$ ribosomal DNA sequencing [21] and biochemical profiles. Sequences were then identified through the use of a mycobacterial species sequence database [22].

2.4. Histopathology. Formalin-fixed tissues were processed and stained with hematoxylin and eosin. Any granulomatous lesions were then stained with a modified Ziehl-Neelson procedure [23]. On the initial animals in the herd that were suspected of having TB based on histopathology, PCR for IS 6110, which identifies $M$. tuberculosis complex bacteria, was performed on formalin-fixed, paraffin embedded tissues. Test protocols followed previously described methods [24].

2.5. Multiantigen Print Immunoassay (MAPIA). MAPIA was performed as previously described by Lyashchenko et al. $[16,25]$. The panel of $M$. bovis antigens included ESAT6, CFP10, MPB59, MPB64, MPB70, MPB83, the 16-kDa protein $(\mathrm{HspX})$, the $38-\mathrm{kDa}$ protein ( $\mathrm{PhoS1} / \mathrm{pstS})$, and Mtb8 (SecE2); three fusion proteins comprising ESAT-6/CFP10, the 16- $\mathrm{kDa}$ protein/MPB83, and F10 (F10 consists of CFP10, secE2, and PhoS1/pstS); two native antigens, M. bovis PPD (B-PPD) and culture filtrate (MBCF).

2.6. CervidTB STAT-PAK Test. The CervidTB STAT-PAK kit (Chembio Diagnostic Systems Inc., Medford, NY, USA) is a lateral-flow test used for rapid detection of antibodies specific to M. tuberculosis complex antigens, ESAT-6, CFP10, and MPB83 $[14,16]$. The device consists of a plastic cassette containing a strip of nitrocellulose membrane impregnated with antigen. The assay employs blue latex microparticles coated with ESAT-6, CFP10, and MPB83. Twenty $\mu \mathrm{L}$ of serum and 3 drops of sample diluent are added sequentially to the sample pad. Results are read visually at $20 \mathrm{~min}$. The presence of a test band of any intensity is considered a positive result whereas no band in the test area is considered a negative result.

2.7. Dual Path Platform (DPP) VetTB Test. The innovative DPP technology utilizes two nitrocellulose strips connected in a " $T$ " shape inside the cassette device [26]. This allows independent delivery of the test sample and antibodydetecting reagent to the assay reaction area, in contrast to the single-strip format used in the CervidTB STAT-PAK test. 
TABLE 1: History of herd and timeline of the outbreak investigation.

\begin{tabular}{|c|c|c|c|}
\hline \multirow{2}{*}{ Action } & \multirow{2}{*}{ Date } & \multicolumn{2}{|l|}{ Number of animals } \\
\hline & & Elk & Fallow deer \\
\hline Original herd size & 2008 & 60 & 50 \\
\hline SCT performed & March 2008 & 50 (all negative) & Not tested \\
\hline Index case detected & January 30, 2009 & 1 & 0 \\
\hline SCT performed ${ }^{\mathrm{a}}$ & February/March 2009 & $42 / 59$ tested ( 3 true positive, $25 / 39$ false negative ${ }^{*}$ ) & Not tested \\
\hline Postmortem examinations ${ }^{\mathrm{b}}$ & June 2009 & 52 & 48 \\
\hline Serum collected & June 2009 & 34/52 examined & 32/48 examined \\
\hline Apparent prevalence ${ }^{\mathrm{c}}$ & June 2009 & 75\% (40/53 TB positive) & $71 \%(34 / 48$ TB positive $)$ \\
\hline
\end{tabular}

The two recombinant antigens, MPB83 and CFP10/ESAT-6 fusion protein, are immobilized on the test strip as separate bands, allowing independent detection of antibody reactivity to each antigen. Five $\mu \mathrm{L}$ of sera is used in the assay, and results are read visually at $20 \mathrm{~min}$. The presence of any of the two test bands was recorded as a positive result while no test band was considered a negative result.

2.8. Test Performance. The two rapid test formats (CervidTB STAT-PAK and DPP VetTB tests) were evaluated for test performance. Data are presented as \% sensitivity ((TP/TP + $\mathrm{FN}) \times 100), \%$ specificity $((\mathrm{TN} / \mathrm{TN}+\mathrm{FP}) \times 100)$, and $\%$ accuracy $((\mathrm{TP}+\mathrm{TN}) /(\mathrm{TP}+\mathrm{FP}+\mathrm{TN}+\mathrm{FN}) \times 100)$, where TP: true positive, TN: true negative, FP: false positive, and FN: false negative.

\section{Results and Discussion}

3.1. Case History, Skin Test, and Necropsy Findings. A timeline providing investigative actions and associated animal numbers is provided in Table 1 . The index case was identified during routine slaughter surveillance inspection (January 30, 2009) when multiple granulomas were identified within the lung and thoracic lymph nodes of an elk carcass originating from a captive cervid farm in Knox County, Nebraska. As part of the USDA's National Bovine Tuberculosis Eradication Program, tissues were submitted to the National Veterinary Services Laboratories for histopathology and culture. Histopathology showed granulomatous pneumonia and lymphadenitis with acid-fast bacilli. PCR of the fixed tissue identified M. tuberculosis complex DNA on February 12, 2009, and M. bovis was subsequently isolated by culture. Approximately 10 months prior, 50/60 elk in this herd had been tested by SCT, and all were negative including the index case. After detection of the M. bovis-infected elk, 42 of the 59 remaining elk were tested by SCT in February/March 2009; 3/42 were SCT-positive, and the 3 SCT positive elk were euthanized and examined postmortem (March 31, 2009). Only 42 were tested by SCT due to animal handling and management issues. All 3 elk had gross and microscopic lesions consistent with TB in the lungs and pleura; PCR confirmed M. tuberculosis complex (April 3, 2009); M. bovis was subsequently isolated by culture in all 3 SCT-positive elk (May 15, 2009). Fallow deer $(n=50)$ in this mixed herd were not tested by SCT primarily due to handling concerns; however, as they had been comingled with M. bovis-infected elk, they were considered TB-exposed. The findings initiated quarantine of the herd and eventual depopulation of all elk and fallow deer on the premises.

Approximately 2 months after $M$. bovis was detected in the 3 SCT-positive elk, the remaining animals within the affected herd were euthanized either on the farm or at a local slaughterhouse. Postmortem examination was completed on 52 of the elk, 39 of which had gross lesions suggestive of TB that was further confirmed by culture and/or histopathology. The majority of elk had tuberculous lesions in multiple tissues, with lung lesions $(n=23$, Figure $1(\mathrm{~b}))$ being the most common, followed by lesions in the thoracic lymph nodes $(n=19)$, pleural lesions $(n=11)$, abdominal lymph node lesions $(n=10)$, and lesions within the head lymph nodes $(n=8)$. On gross inspection, the TB lesions appeared as $1 \mathrm{~mm}$ to $10 \mathrm{~cm}$ inflammatory masses with a yellow liquid necrotic center (Figure 1(a)) or as masses that were solid on cut surface and frequently mineralized. Within the 48 fallow deer in which postmortem examination was completed, 34 fallow deer had gross lesions suggestive of $\mathrm{TB}$ that were confirmed by culture and/or histopathology. The majority of fallow deer had lesions in multiple tissues, with lesions in the thoracic lymph nodes being most common $(n=21)$, followed by lesions in the abdominal lymph nodes $(n=18)$, lesions in the head lymph nodes $(n=10)$, and lung lesions $(n=6)$. The lesions were highly variable in size, and the majority had the gross appearance of an abscess. In general, gross lesions were more severe in elk as compared to fallow deer. Based on histopathology and/or culture results, the prevalence of TB was estimated at $\sim 71-75 \%$ (fallow deer: 34/48; elk: 40/53, including the index case from January 30 , 2009). 


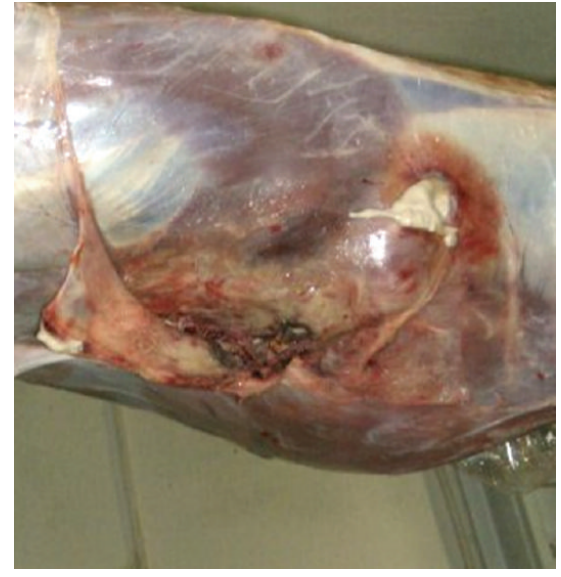

(a)

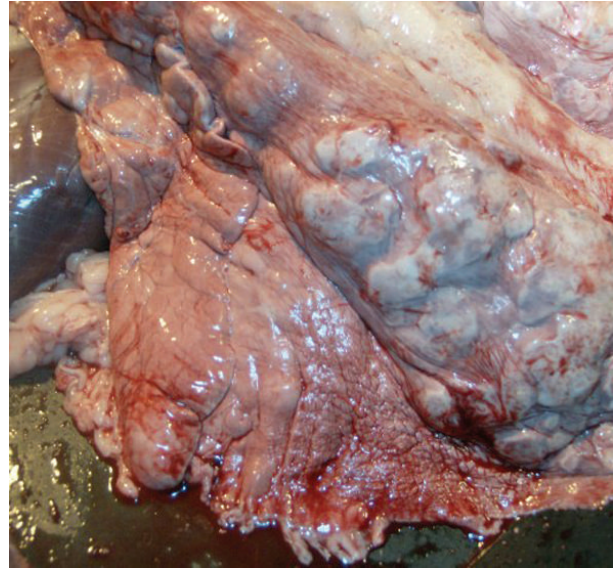

(b)

Figure 1: Gross lesions in elk. (b) demonstrates a tuberculous thoracic lesion that has eroded through the body wall. (a) demonstrates coalescing, multinodular, pulmonary granulomas. Orientation of both photographs: cranial to the left and dorsal to the top.

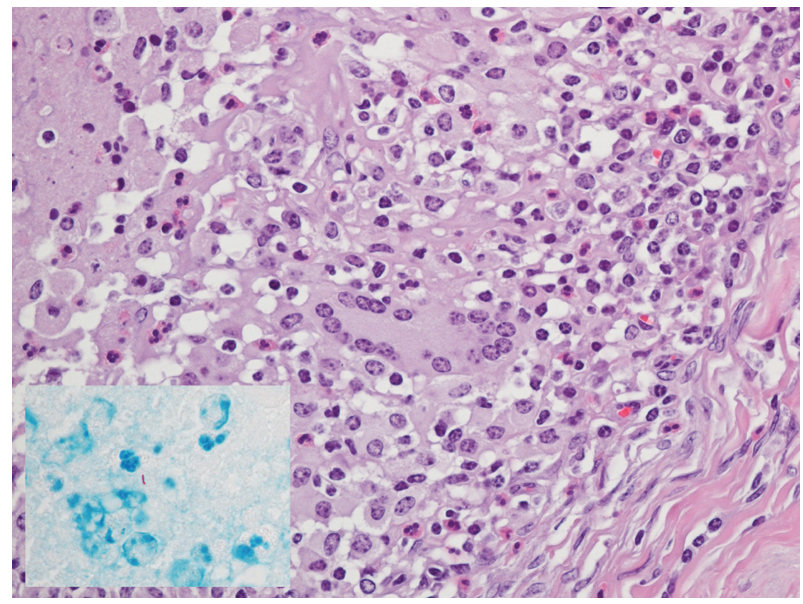

FIGURE 2: Microscopic lesions in elk. The image is a photograph of a representative pulmonary granuloma in an elk (hematoxylin and eosin stain). Note the large number of neutrophils and the single multinucleate giant cell in the center of the image. The inset image demonstrates an acid-fast bacillus (Ziehl-Neelson stain) in a pool of proteinaceous fluid, necrotic cell debris, and degenerate neutrophils.

Microscopically, the TB lesions consisted of either granulomas or pyogranulomas with rare to large numbers of acidfast bacilli (Figure 2). The histological lesions in infected animals were quite variable between animals as previously described for TB in cervid species [27-30]. Briefly, there were three general subtypes of lesions in both the elk and the fallow deer. The first type of lesion consisted predominantly of variably sized foci containing predominantly macrophages, and epithelioid macrophages, along with low numbers of multinucleated (Langhans) giant cells. Lymphocytes and plasma cells were intermixed with small amounts of fibrous connective tissue and as small lymphocytic aggregates. These lesions were often multifocal to coalescing, but lacked a central area of necrotic cell debris. A second type of lesion was similar to the classical tubercles commonly observed in cattle infected with M. bovis. These lesions contained a central core of caseous necrosis with variable amounts of mineralized necrotic debris. The margins of the lesion were composed of a mixture of macrophages, epithelioid macrophages, lymphocytes, plasma cells, and occasional multinucleated (Langhans) giant cells. There was often a thin fibrous band at the outermost margins. The third type of lesion also had a large central area of necrosis; however, the central core contained large numbers of neutrophils and degenerative neutrophils. The neutrophils extended into a surrounding rim of macrophages, epithelioid macrophages, lymphocytes, plasma cells, and fibrous connective tissue. When pyogranulomas were identified in lung tissue, the adjacent bronchioles and alveoli were frequently filled with proteinaceous fluid, neutrophils, and degenerate neutrophils.

M. bovis was isolated by culture of lesions in 34/59 elk and 32/50 fallow deer. Based upon culture alone, the prevalence of TB was estimated at $58-64 \%$ for elk and fallow deer, respectively. Multiple mycobacterial isolates were recovered from one elk and two fallow deer; $M$. bovis was recovered from all three, $M$. thermoresistibile was also recovered from the elk, and $M$. avium complex from the two fallow deer. M. avium was also recovered from one fallow deer in which $M$. bovis was not recovered. All $M$. bovis isolates from this outbreak had the same spoligotype, octal code 666773677777600 . This spoligotype was initially encountered in an isolate recovered from an elk residing in New York state in 1991 and has since sporadically resurfaced in cervid herds throughout the USA (Robbe-Austerman and Harris, unpublished observations).

Twenty-five of 39 elk which tested negative on SCT approximately 3 months prior to necropsy had microscopic lesions consistent with TB and containing acid-fast bacilli and/or $M$. bovis isolated by culture of lesions. Considering 


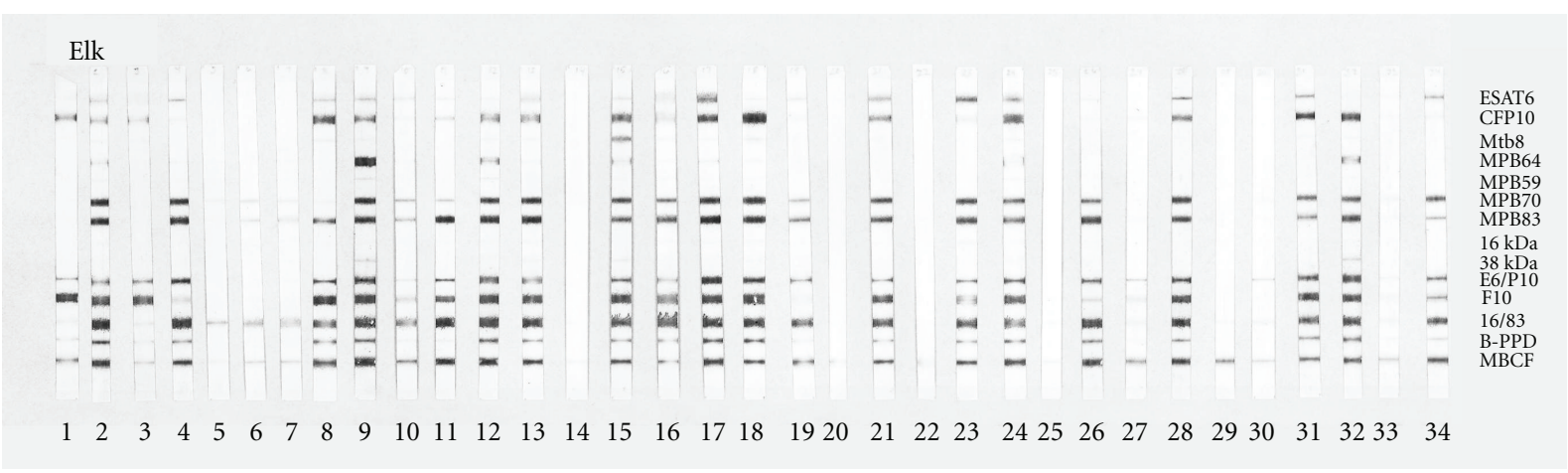

(a)

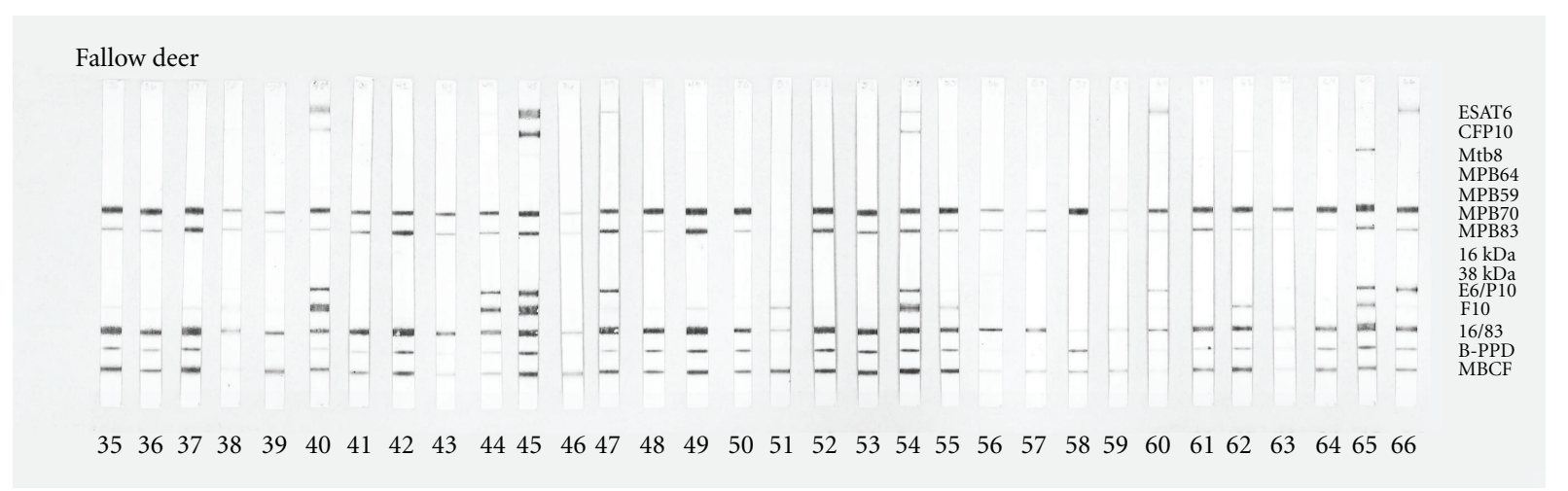

(b)

FIgURE 3: Antibody responses to recombinant and native antigens detected in elk (a) and fallow deer (b) using a multiantigen print immunoassay. Numbers in lower margins indicate unique animal identification designations. Antigens printed on nitrocellulose are indicated in the right margin. E6/P10 refers to a fusion protein of ESAT-6 and CFP10. 16/83 refers to a fusion protein of the 16kDa protein and MPB83. F10 is a fusion protein consisting of CFP10, secE2, and PhoS1/pstS. B-PPD refers to M. bovis-derived purified protein derivative. MBCF refers to $M$. bovis-derived culture filtrate antigen.

the initial 3 that were identified as SCT responsive, the sensitivity of SCT in elk within this herd was unexpectedly low (3/28). As all elk were considered exposed, the specificity of SCT was not possible to determine.

3.2. MAPIA. Serum was collected from 34 elk (all SCT negative $\sim 3$ months prior) and 32 fallow deer (not tested by SCT) from the Nebraska herd-all 66 animals were considered tuberculous based on histopathology and culture results. Serum was not collected from the 3 SCT-positive elk. Extensive reactivity with single $M$. bovis antigens (i.e., ESAT-6, CFP10, MPB70, and MPB83), fusion proteins (i.e., F10, ESAT-6/CFP10, and the $16-\mathrm{kDa}$ protein/MPB83 fusions), and complex antigens (i.e., B-PPD and MBCF) was detected with sera from elk (Figure 3). Lesser frequency, but detectable reactivity was seen with Mtb8 and MPB64 antigens. Minimal to no reactivity was observed with MPB59, $16-\mathrm{kDa}$ protein, and $38-\mathrm{kDa}$ protein.

Antibody responses were generally less robust in fallow deer as compared to those in elk (Figure 3). Predominant antigen recognition patterns were similar between elk and fallow deer, although less frequent reactivity to ESAT-6 and CFP10 antigens was detected with sera from fallow deer. MAPIA was positive in $28 / 34(82 \%)$ tuberculous elk and in $31 / 32$ (97\%) tuberculous fallow deer. The rates of antibody reactivity as well as antigen recognition patterns found by MAPIA in the present study appear to be in agreement with the previous report on elk experimentally infected with $M$. bovis [14].

3.3. CervidTB STAT-PAK and DPP VetTB Tests. Two rapid assays were evaluated for test sensitivity with sera from this herd ( $n=34$ elk, $n=32$ fallow deer; all 66 were TB-positive). Additionally, sera from noninfected elk $(n=141)$ and fallow deer $(n=107)$ originating from known TB-free herds were obtained from the APHIS, NVSL bovine TB serum bank for evaluation of test specificity. Results are presented in Table 2 (elk) and Table 3 (fallow deer). Findings demonstrate high sensitivity and specificity of both test formats with elk (se: 79 to $82 \%$, sp: 93 to $98 \%$ ) and fallow deer (se:91\%, sp:91 to $99 \%)$. The DPP VetTB assay was more accurate than CervidTB STAT-PAK test (94-97\% versus 91\%, resp.) in 
TABLE 2: Diagnostic performance of serological tests in elk.

\begin{tabular}{lccc}
\hline Assay & Sensitivity $^{\mathrm{a}}$ & Specificity $^{\mathrm{b}}$ & Accuracy \\
\hline CervidTB STAT-PAK & $82 \%(28 / 34)$ & $93 \%(131 / 141)$ & $91 \%(159 / 175)$ \\
DPP VetTB & $79 \%(27 / 34)$ & $98 \%(138 / 141)$ & $94 \%(165 / 175)$ \\
\hline
\end{tabular}

${ }^{a}$ Elk were considered positive for bovine TB if either M. bovis was isolated upon culture of lesions or mycobacteriosis compatible lesions were detected upon microscopic examination of gross lesions.

${ }^{\mathrm{b}}$ Sera from noninfected and SCT-negative elk were obtained from the APHIS, NVSL bovine TB serum bank for evaluation of test specificity.

TABLE 3: Diagnostic performance of serological tests in fallow deer.

\begin{tabular}{lccr}
\hline Assay & Sensitivity $^{\mathrm{a}}$ & Specificity $^{\mathrm{b}}$ & Accuracy \\
\hline CervidTB STAT-PAK & $91 \%(29 / 32)$ & $91 \%(97 / 107)$ & $91 \%(126 / 139)$ \\
DPP VetTB & $91 \%(29 / 32)$ & $99 \%(106 / 107)$ & $97 \%(135 / 139)$ \\
\hline
\end{tabular}

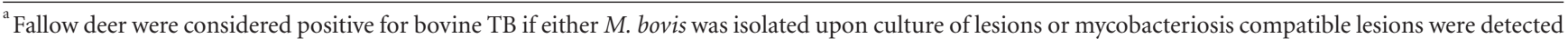
upon microscopic examination of gross lesions.

bSera from noninfected and SCT-negative fallow deer were obtained from the APHIS, NVSL bovine TB serum bank for evaluation of test specificity.

both cervid species. These results support the serodiagnostic potential for CervidTB STAT-PAK and DPP VetTB tests previously shown for several cervid species [13-16].

3.4. Seroreactivity of Individual Animals with Each Test. In general, the seroreactivity of individual animals between test formats was similar. With TB-infected elk sera, all 3 tests (i.e., CervidTB STAT-PAK, DPP VetTB, and MAPIA) were positive with 27/34 samples. All 3 tests were negative for TB-infected elk numbers 5, 14, 20, 22, 25, and 29. The DPP VetTB test was also negative with TB-infected elk number 33. With TBinfected fallow deer sera, all 3 tests were positive with 29/32 samples. CervidTB STAT-PAK and DPP VetTB tests were negative with TB-infected fallow deer numbers 58 and 59 and all three tests were negative with fallow deer number 51. These findings demonstrate high concordance between the serological tests used in the present study. MAPIA band intensities (indicative of levels of antibody reactivity with antigens) were generally greater in elk than those observed in fallow deer (Figure 3), presumably due to more advanced disease or prior injection of PPD for SCT in elk. These results suggest that the high-prevalence of $M$. bovis infection found in this mixed cervid herd in early 2009 had originally occurred in elk. The elk may have failed to produce an SCT response in March 2008 (as also observed in February/March 2009), presumably transmitting the infection to fallow deer upon co-mingling with elk. A lesser severity of disease and lower levels of $M$. bovis-specific antibodies support the hypothesis that the infection initiated in the elk herd prior to the fallow deer. However, disease progression and development of $M$. bovis-specific responses likely differ, potentially remarkably, between these two host species; thus, it is not clear as to which species was first infected.

Unique findings from this case report include unusually high prevalence of TB in this herd and the unexpectedly low sensitivity of SCT in elk. While purely speculative, the high prevalence of disease may be due to an extended course of infection as indicated by the severity of lesions. Reasons for the SCT failure could be explained by anergy of cell-mediated immune responses associated with advanced stages of disease or specific host factors. Anecdotal evidence supports the potential for a predominance of antibody responses by elk to various intracellular pathogens (Waters, unpublished observations and Steven Olsen, personal communication). Another possibility, although less likely, is that disease progression within the elk occurred very rapidly, developing some time after SCT. However, prior studies with experimental TB infection in cervids would not support such a rapid progression of disease $[14,24,30]$. Further research is warranted to characterize immune responses by elk and fallow deer to various intracellular pathogens, especially M. bovis.

Limitations of the present study include that not all animals were evaluated by SCT, necropsy, culture, and serology; samples for histology and culture were only collected from animals with gross lesions; SCT was performed 3 months prior to necropsy to determine disease status; serologic analysis for determination of sensitivity was only performed on animals with gross lesions.

\section{Conclusions}

Present findings demonstrate (1) an example of an unusually high prevalence $(\sim 71-75 \%)$ and severity of infection in a captive elk and fallow deer herd in Nebraska, USA, (2) unexpectedly poor sensitivity of SCT for detection tuberculous elk in this herd, and (3) opportunities for use of serology for the rapid detection of M. bovis infection in captive elk and fallow deer.

\section{Acknowledgments}

The authors greatly appreciate the support of (1) Drs. William Stump (USDA APHIS VS-Nebraska) and Brian Archer (NVSL, APHIS, USDA, Kansas) with sample collection, preparations and shipping as well as record keeping; (2) Drs. W. Hutcheson (USDA, FSIS) and Martha Elmore 
(NVSL, APHIS, USDA, Nebraska) for the postmortem examinations at the slaughter plant and on the farm, respectively; (3) numerous others from NVSL, APHIS, USDA, Nebraska Department of Agriculture, and the Nebraska Game and Parks Commission for organization and assistance with the investigation; (4) Claudia Quinn for excellent technical assistance with serological testing. USDA is an equal opportunity provider and employer. Mention of trade names or commercial products in this publication is solely for the purpose of providing specific information and does not imply recommendation or endorsement by the U.S. Department of Agriculture.

\section{References}

[1] J. F. T. Griffin and C. G. Mackintosh, "Tuberculosis in deer: perceptions, problems and progress," Veterinary Journal, vol. 160, no. 3, pp. 202-219, 2000.

[2] G. W. De Lisle, C. G. Mackintosh, and R. G. Bengis, "Mycobacterium bovis in free-living and captive wildlife, including farmed deer," OIE Revue Scientifique et Technique, vol. 20, no. 1, pp. 86-111, 2001.

[3] M. A. Essey and M. A. Koller, "Status of bovine tuberculosis in North America," Veterinary Microbiology, vol. 40, no. 1-2, pp. 15-22, 1994.

[4] D. J. O’Brien, S. M. Schmitt, S. D. Fitzgerald, D. E. Berry, and G. J. Hickling, "Managing the wildlife reservoir of Mycobacterium bovis: the Michigan, USA, experience," Veterinary Microbiology, vol. 112, no. 2-4, pp. 313-323, 2006.

[5] T. L. Whiting and S. V. Tessaro, "An abattoir study of tuberculosis in a herd of farmed elk," Canadian Veterinary Journal, vol. 35, no. 8, pp. 497-501, 1994.

[6] A. Fanning and S. Edwards, "Mycobacterium bovis infection in human beings in contact with elk (Cervus elaphus) in Alberta, Canada," Lancet, vol. 338, no. 8777, pp. 1253-1255, 1991.

[7] T. J. Bucholz, Bovine TB Strain Confirmed In Michigan Hunter: Hunters Reminded To Wear Gloves When Cleaning Game, Michigan Department of Community Health, http://www.michigan.gov/mdch/0,1607,7-132-8347-107460M,00.html.

[8] M. J. Wilkins, J. Meyerson, P. C. Bartlett et al., "Human Mycobacterium bovis infection and bovine tuberculosis outbreak, Michigan, 1994-2007," Emerging Infectious Diseases, vol. 14, no. 4, pp. 657-660, 2008.

[9] C. E. Massengill, "Report of the committee on tuberculosis," in Proceedings of the 109th United States Animal Health Association Annual Meeting, vol. 109, pp. 679-718, Hershey, $\mathrm{Pa}$, USA, 2005.

[10] C. E. Massengill, "Report of the committee on tuberculosis," in Proceedings of the 108th United States Animal Health Association Annual Meeting, vol. 108, pp. 581-616, Greensboro, NC, USA, 2004.

[11] W. C. Hench, "Current status of the US bovine tuberculosis eradication program fiscal year," in Proceedings of the 111th United States Animal Health Association Annual Meeting, vol. 111, pp. 747-752, Reno, Nev, USA, 2007.

[12] K. M. Connell, "Bovine tuberculosis surveillance in U.S. livestock fiscal year 2009," in Proceedings of the 113th United States Animal Health Association Annual Meeting, vol. 113, pp. 650-653, San Diego, Calif, USA, 2009.

[13] B. M. Buddle, T. Wilson, M. Denis et al., "Sensitivity, specificity, and confounding factors of novel serological tests used for the rapid diagnosis of bovine tuberculosis in farmed red deer (Cervus elaphus)," Clinical and Vaccine Immunology, vol. 17, no. 4, pp. 626-630, 2010.

[14] N. P. Harrington, O. P. Surujballi, J. F. Prescott et al., "Antibody responses of cervids (Cervus elaphus) following experimental Mycobacterium bovis infection and the implications for immunodiagnosis," Clinical and Vaccine Immunology, vol. 15, no. 11, pp. 1650-1658, 2008.

[15] R. Jaroso, J. Vicente, M. P. Martín-Hernando et al., "Antemortem testing wild fallow deer for bovine tuberculosis," Veterinary Microbiology, vol. 146, no. 3-4, pp. 285-289, 2010.

[16] K. P. Lyashchenko, R. Greenwald, J. Esfandiari et al., "Animalside serologic assay for rapid detection of Mycobacterium bovis infection in multiple species of free-ranging wildlife," Veterinary Microbiology, vol. 132, no. 3-4, pp. 283-292, 2008.

[17] W. R. Waters, M. V. Palmer, J. P. Bannantine et al., "Antigen recognition by serum antibodies in white-tailed deer (Odocoileus virginianus) experimentally infected with Mycobacterium bovis," Clinical and Diagnostic Laboratory Immunology, vol. 11, no. 5, pp. 849-855, 2004.

[18] W. R. Waters, M. V. Palmer, J. P. Bannantine et al., "Antibody responses in reindeer (Rangifer tarandus) infected with Mycobacterium bovis," Clinical and Diagnostic Laboratory Immunology, vol. 12, no. 6, pp. 727-735, 2005.

[19] United States Department of Agriculture: Animal and Plant Health Inspection Service, Veterinary Services. Bovine tuberculosis eradication uniform methods and rules. U.S. Government Printing Office, Washington, DC, USA, pp.19-34, 1999.

[20] N. Hines, J. B. Payeur, and L. J. Hoffman, "Comparison of the recovery of Mycobacterium bovis isolates using the BACTEC MGIT 960 system, BACTEC 460 system, and Middlebrook 7H10 and 7H11 solid media," Journal of Veterinary Diagnostic Investigation, vol. 18, no. 3, pp. 243-250, 2006.

[21] P. Kierschner and E. C. Bottger, "Species identification of Mycobacteria using rDNA sequencing," in Methods in Molecular Biology, Mycobacteria Protocols, T. Parish and N. G. Stoker, Eds., vol. 101, Humana Press Inc., Totowa, NJ, USA, 1998.

[22] D. Harmsen, S. Dostal, A. Roth et al., "RIDOM: comprehensive and public sequence database for identification of Mycobacterium species," BMC Infectious Diseases, vol. 3, article 26, 2003.

[23] A. Penrod, "Modified Ziehl-Neelsen staining procedure," in Standard Operating Procedure SOP-PL-38.05, National Veterinary Services Laboratories Ames (IA), 2010.

[24] M. V. Palmer, W. R. Waters, T. C. Thacker, W. C. Stoffregen, and B. V. Thomsen, "Experimentally induced infection of reindeer (Rangifer tarandus) with Mycobacterium bovis," Journal of Veterinary Diagnostic Investigation, vol. 18, no. 1, pp. 52-60, 2006.

[25] K. P. Lyashchenko, M. Singh, R. Colangeli, and M. L. Gennaro, "A multi-antigen print immunoassay for the development of serological diagnosis of infectious diseases," Journal of Immunological Methods, vol. 242, no. 1-2, pp. 91-100, 2000.

[26] R. Greenwald, O. Lyashchenko, J. Esfandiari et al., "Highly accurate antibody assays for early and rapid detection of tuberculosis in african and asian elephants," Clinical and Vaccine Immunology, vol. 16, no. 5, pp. 605-612, 2009.

[27] E. B. Rohonczy, A. V. Balachandran, T. W. Dukes et al., "A comparison of gross pathology, histopathology, and mycobacterial culture for the diagnosis of tuberculosis in elk (Cervus elaphus)," Canadian Journal of Veterinary Research, vol. 60, no. 2, pp. 108-114, 1996.

[28] J. C. Rhyan and D. A. Saari, "A comparative study of the histopathologic features of bovine tuberculosis in cattle, fallow 
deer (Dama dama), sika deer (Cervus nippon), and red deer and elk (Cervus elaphus)," Veterinary Pathology, vol. 32, no. 3, pp. 215-220, 1995.

[29] J. C. Rhyan, D. A. Saari, E. S. Williams, M. W. Miller, A. J. Davis, and A. J. Wilson, "Gross and microscopic lesions of naturally occurring tuberculosis in a captive herd of wapiti (Cervus elaphus nelsoni) in Colorado," Journal of Veterinary Diagnostic Investigation, vol. 4, no. 4, pp. 428-433, 1992.

[30] M. V. Palmer, W. R. Waters, and D. L. Whipple, "Lesion development in white-tailed deer (Odocoileus virginianus) experimentally infected with Mycobacterium bovis," Veterinary Pathology, vol. 39, no. 3, pp. 334-340, 2002. 

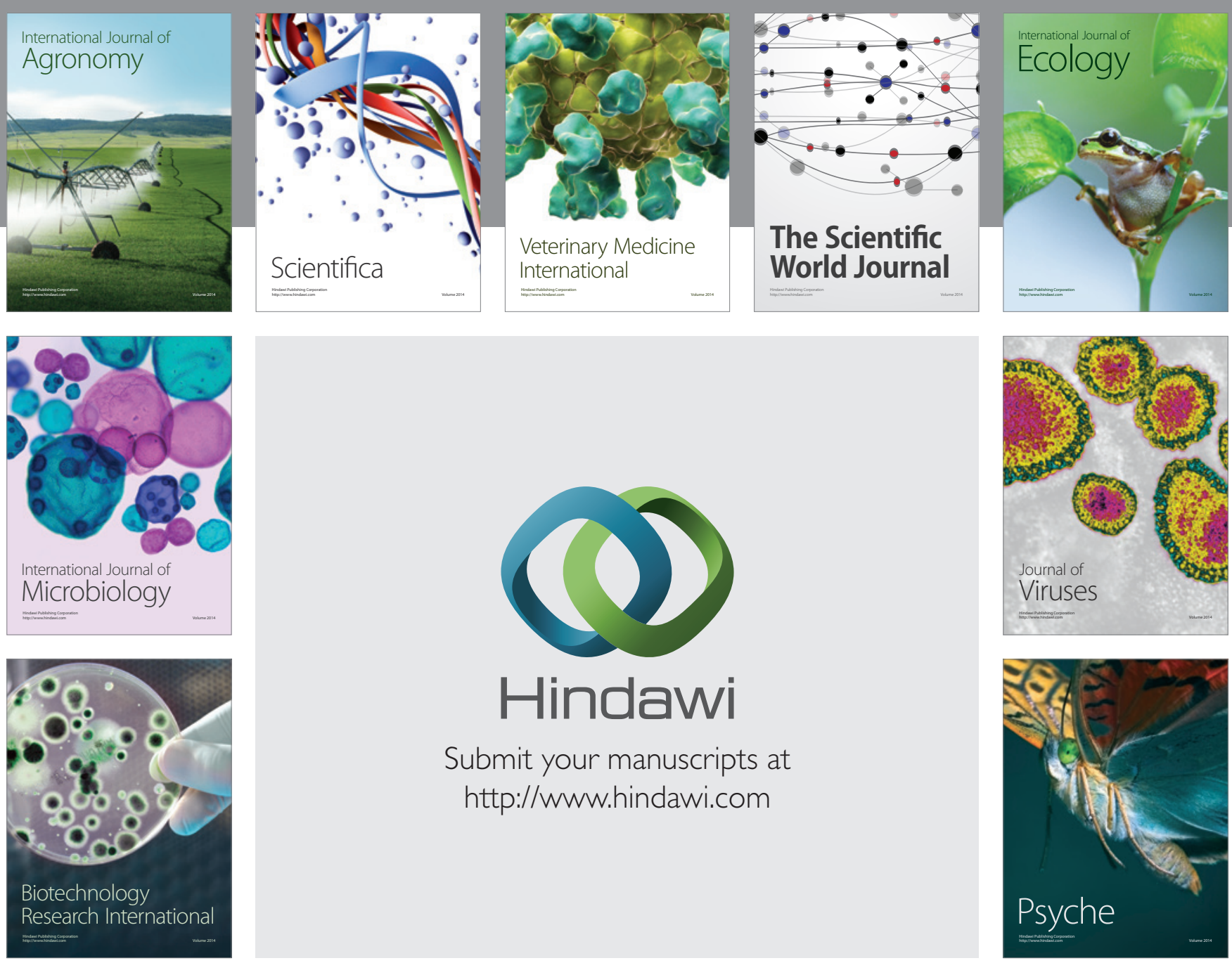

Submit your manuscripts at

http://www.hindawi.com
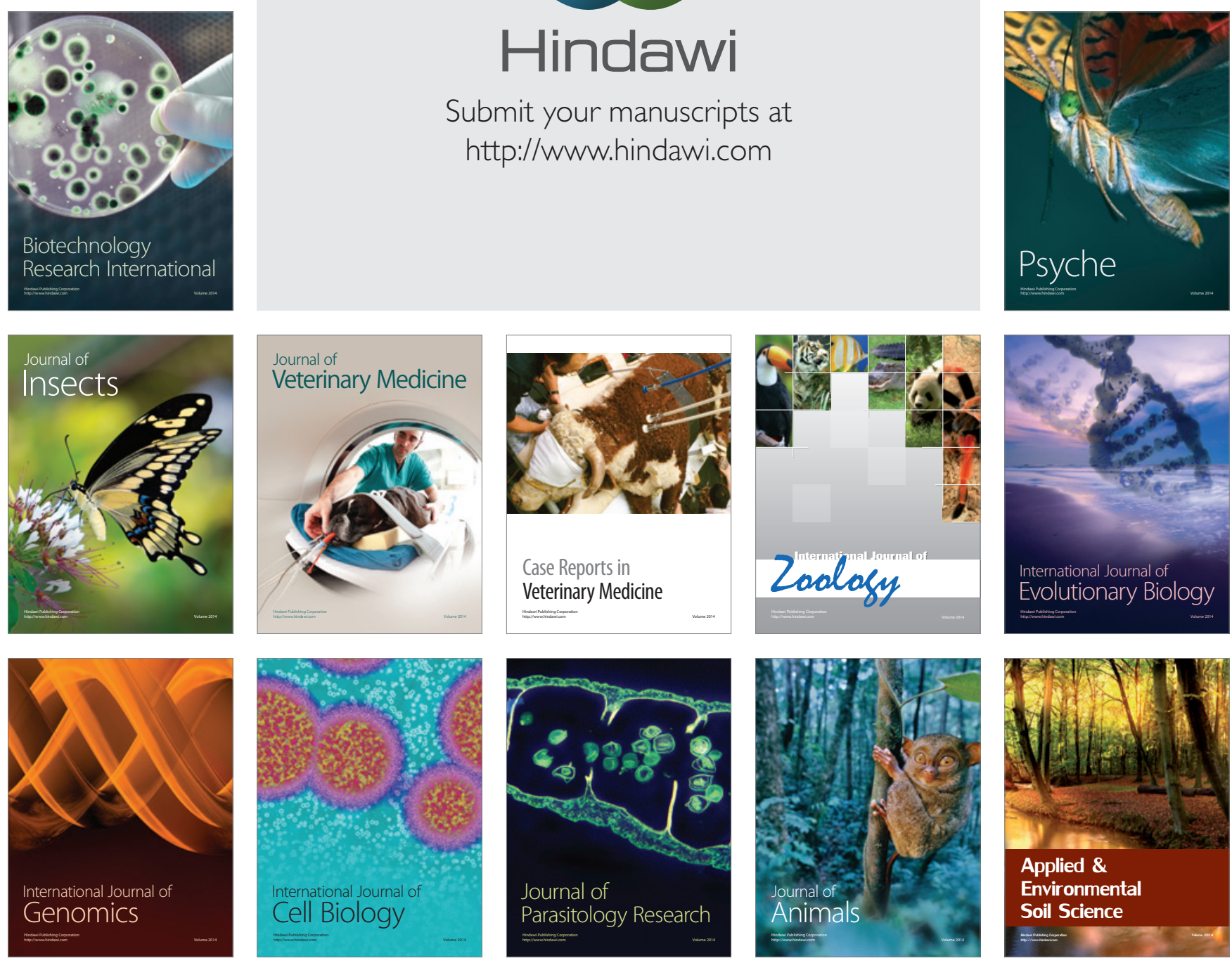